\title{
A generic approach for mechano-chemical reactions between carbonnanotubes of different functionalities
}

\author{
Mohamad A Kabbani ${ }^{1 * *}$, Chandra Sekhar Tiwary ${ }^{1}$, Anirban Som ${ }^{2 *}$, K. R. Krishnadas ${ }^{2 *}$, Pedro A. \\ S. Autreto ${ }^{1,3}$, Sehmus Ozden $^{1}$, Kunttal Keyshar ${ }^{1}$, Ken Hackenberg ${ }^{1}$, Alin Christian Chipara ${ }^{1}$, \\ RobertVajtai $^{1}$,Ahmad T Kabbani ${ }^{4}$,Thalappil Pradeep ${ }^{2 * *}$ and Pulickel M. Ajayan ${ }^{1,2^{* *}}$ \\ ${ }^{1}$ Department of Materials Science and Nano Engineering, Rice University, Houston, Texas 77005, USA \\ ${ }^{2}$ DST Unit of Nanoscience and Thematic Unit of Excellence, Department of Chemistry, Indian Institute of \\ Technology Madras, Chennai 600 036, India \\ ${ }^{3}$ Applied Physics Department, State University of Campinas, Campinas-SP, 13083-959, Brazil \\ ${ }^{4}$ Natural Science department, Lebanese American University, Beirut: 11022801 Lebanon \\ * Equal contribution
}

\begin{abstract}
:
Here, we report similar reactions between nanotubes carrying functionalities, namely carbon nanotubes ( CNTs) with the acyl chloride/hydroxyl and amine/carboxylic functionalities directly attached to their surfaces, resulting in the formation ofchemically modified graphene products. The reaction is spontaneous and is facilitated by simple grinding of the reactants. The new solidstate reactions have been confirmed using different spectroscopic and electron microscopy techniques.
\end{abstract}

Keywords:Mechanochemical reactions, carbon-nanotubes, unzipping mechanism,graphene.

Corresponding author.

Tel:713-348-5904, Fax: 713-348-5423,

Email: mak8@ rice.edu (MAK), pradeep@iitm.ac.in (TP), ajayan@ rice.edu (PMA) 


\section{Introduction}

Nanoscale materials are attractive due to their unique size, large surface area, chemical and physical properties. Great advancements have been made in the last decade in the synthesis of nanoparticles, which led to new innovative applications in various fields such as catalysis, chemical sensing, photonics, electronic devices and drug delivery [1-8]. Although functionalization has been extensively used to overcome limitations, such as poor solubility, reactivity and processing, by conjugating these particles to different chemical moieties such as drug molecules, polymers or organic molecules, the reactions between differently functionalized nanoparticles have been given very little attention. Especially important, is how do the attributes of these particles reflect on the rate of the reaction and how do the nanoscopic reactivities compare to that of the bulk molecular one. The most remarkable chemical reaction reported between nanoparticles is the dimerization of fullerene molecules inside carbon nanotubes 'peapods' [9-10]. In this type of confinement reaction, the degrees of freedom are restricted to translational motion in one dimension. Encapsulation of the $\mathrm{C}_{60} \mathrm{~s}$ in the CNT peapod structure has been reported to cause a decrease of fullerenes intermolecular distance by 3-4 \% than in bulk crystals. Structure optimization techniques indicates that the net energy gain associated with the encapsulation of the $\mathrm{C}_{60} \mathrm{~s}$ gives rise to a capillary force with an effective pressure of the order of GPa that will give rise to a strain on the CNT wall. Other than the confinement peapod fullerene/CNT dimerization reaction, no reaction has been reported between nanoparticles of different functionalities. Although an abundance of both top-bottom and bottom-up synthetic strategies have evolved in the last decade for the production of graphene [11-25], none of them considered the solid-state reaction between CNTs of different functionalizations. 
We have recently reported the unzipping of MWCNTs via a one-pot room temperature solidstate mechano-chemical reaction between MWCNT-COOH and MWCNT-OH.The reaction involves hydrogen bond mediated proton transfer step that is followed by two elimination steps: water condensation and decarboxylation [26]. To test this type of solid-statedouble eliminations scheme, we here, report two new modifications of mechano-chemical reactions of differently functionalized MWCNTs,namely MWCNT-COCl/MWCNT-OH and MWCNT-NH $2 / \mathrm{MWCNT}_{-}$ $\mathrm{OH}$ reactions, Fig.1.

Reactions are facilitated by manual grinding of equal weights of the functionalized CNTs as described in our previous report [26]. The two new modifications together with the previously reported one are summarized in equations 1-3

MWCNT-COOH + MWCNT-OH $\rightarrow \mathrm{G}+\mathrm{G}^{\prime}+\mathrm{CO}_{2}+\mathrm{H}_{2} \mathrm{O}$

MWCNT-NH $2+\mathrm{MWCNT}-\mathrm{COOH} \rightarrow \mathrm{G}+\mathrm{G}^{\prime}+\mathrm{NH}_{3}+\mathrm{CO}_{2}$

$\mathrm{MWCNT}-\mathrm{COCl}+\mathrm{MWCNT}-\mathrm{OH} \rightarrow \mathrm{G}+\mathrm{G}^{\prime}+\mathrm{CO}_{2}+\mathrm{HCl}$

$\mathrm{G}$ and $\mathrm{G}$ 'are the graphene types derived from the two differently functionalized CNTs.

In reaction 2,the unzipping double elimination is mediated by hydrogen-bond formation, followed by proton transfer from the $\mathrm{COOH}$ group to the $\mathrm{NH}_{2}$, double elimination of $\mathrm{NH}_{3}$ and $\mathrm{CO}_{2}$,formation of CNTs ion pair and consequent unzipping of CNTs by the heat of the exothermic reaction [26], while the double elimination in reaction 3 proceeds via the condensation of $\mathrm{HCl}$, decarboxylation, CNTs ion pair formation and CNTs unzipping [26].Each of the above reaction can be considered as a double elimination reaction as summarized in Table.1.Graphene products as well as the double elimination products of the new 
mechanochemical modifications are confirmed using spectral techniques and electron microscopy ones.

\section{Results and Discussion}

ATR-IR of the solid-state reaction product in the reaction mixture MWCNT-COOH/MWCNT$\mathrm{NH}_{2}$, (Fig2a) reveals almost complete absence of the strong doublet band at about $3000 \mathrm{~cm}^{-1}$ due to the N-H stretching mode as well as absence of broad bands in the region $2500-3000 \mathrm{~cm}^{-1}$ due to $\mathrm{O}-\mathrm{H}$ stretching in the MWCNT-COOH/MWCNT-NH 2 mixture in agreement with water and ammonia condensation reaction. Also, the intensity of the band at $1680 \mathrm{~cm}^{-1}$ due to the carbonyl band of the carboxylic group diminishes significantly with appearance of the adsorbed $\mathrm{CO}_{2}$ and $\mathrm{NH}_{3}$ bands at 2370 and $3510 \mathrm{~cm}^{-1}$, respectively [27]. Compatible with this conclusion is the disappearance of the two strong bands due the C-N stretching in the region $1180-1360 \mathrm{~cm}^{-1}$ mode and absence the broad band at about $900 \mathrm{~cm}^{-1}$ due to $\mathrm{N}-\mathrm{H}$ bending in the graphene product of the reaction. Similarly, the conjugated carbonyl band of the acyl chloride at $1750 \mathrm{~cm}^{-1}$ and broad $\mathrm{OH}$ stretching bands of the CNT are almost absent in the product of the solid-state reaction of MWCNT-COCl and MWCNT-OH with the $\mathrm{C}=\mathrm{C}$ band at $1600 \mathrm{~cm}^{-1}$ of the graphene becoming dominant (Fig.2a-b).

In the C1s XPS of the MWCNTs, the signal at $291 \mathrm{eV}$ due to the carbonyl group of the acyl and carboxylic MWCNTs disappears upon grinding while the signal at $284.6 \mathrm{eV}$ (Fig. 2c-d) due to $\mathrm{C}=\mathrm{C}$ becomes the dominant signal [28]. This is strong evidence in favor of graphene formation and therefore doubles condensation reactions in the MWCNTCOCl/MWCNT-OH and $\mathrm{MWCNTNH}_{2} / \mathrm{MWCNT}-\mathrm{COOH}$ reactive mixtures. In addition, according to XPS, chlorine content in reaction 3 drops from $0.195 \%$ before grinding to $0.056 \%$ in the graphene product 
giving rise to a yield of about $70 \%$.This is accompanied by a drop in the oxygen content from 0.304 in the reaction mixture to $0.132 \%$ (XPS data, Supplementary Table 1).In the $\mathrm{MWCNTNH}_{2} / \mathrm{MWCNT}-\mathrm{COOH}$ nitrogen content drops from $0.300 \%$ in the amine CNT to $0.0375 \%$ in the graphene product-giving rise to $75 \%$ yield reaction.

Raman spectroscopy is a quick, easy and powerful tool that can be used for structural as well as quality characterization of the graphene products in the mechanochemical reactions. The intensity ratio of the $\mathrm{D}$ and $\mathrm{G}$ bands, $\mathrm{I}_{\mathrm{D}} / \mathrm{I}_{\mathrm{G}}$, is commonly used to evaluate the quality of the graphene material. The $\mathrm{I}_{\mathrm{D}} / \mathrm{I}_{\mathrm{G}}$ for the graphene products are 0.23 and 0.41 for the MWCNTCOCl/MWCNT-OH and MWCNTCOOH/MWCNT-NH ${ }_{2}$ combinations respectively, Fig.2e-f. The low value of the graphene due to the graphene product in the former is consistent with 1-3 layers graphene quality obtained via the CVD method where the upper limit of $\mathrm{I}_{\mathrm{D}} / \mathrm{I}_{\mathrm{G}}$ was found to be $0.3[19]$.This result is consistent with the $\mathrm{I}_{2 \mathrm{D}} / \mathrm{I}_{\mathrm{G}}$ ratio whose value of $\sim 2$ hallmarks the low layers graphene quality. On the other hand both ratios of $\mathrm{I}_{\mathrm{D}} / \mathrm{I}_{\mathrm{G}}$ $(0.41)$ and $\mathrm{I}_{2 \mathrm{D}} / \mathrm{I}_{\mathrm{G}}(0.95)$ for the graphene obtained via the $\mathrm{NH} 2 / \mathrm{COOH}$ are consistent with graphene of more than three layers graphene according to the CVD scale. The enhanced numbers of layers could be related to greater tendency for the $\mathrm{NH}_{2}$ and $\mathrm{COOH}$ groups to hydrogen bond. Yields of the graphene obtained via different solid-state reactions are summarized in table 2.

In order to confirm the formation of $\mathrm{HCl}$ during the reaction between $\mathrm{CNT}-\mathrm{COCl}$ and $\mathrm{CNT}-\mathrm{OH}$, the gaseous products formed during the grinding of these CNTs (in a mortar-pestle set up) were passed continuously into an aqueous $\mathrm{AgNO}_{3}(10 \mathrm{mM})$ solution by using high pure $\mathrm{N}_{2}(99.9 \%)$ as carrier gas. Schematic of is given in Fig. 3a. After 30 minutes of passing the gas mixture with continuous grinding, a white turbidity appeared in the $\mathrm{AgNO}_{3}$ solution. This turbid solution was drop casted for SEM imaging (Fig. 3b) and EDAX elemental analysis (Fig. 3c), which showed the presence of $\mathrm{AgCl}$ particles confirming evolution of $\mathrm{HCl}$ gas in reaction between $\mathrm{CNT}-\mathrm{COCl}$ 
and $\mathrm{CNT}-\mathrm{OH}$. A control experiment with only the $\mathrm{CNT}-\mathrm{COCl}$ was conducted using the same set-up for which formation of $\mathrm{AgCl}$ was not observed.

As for reaction 3, evolution of gaseous ammonia during the reaction between $-\mathrm{COOH}$ and $\mathrm{NH}_{2}$ functionalized MWCNTs was confirmed through a mass spectrometrometric detection procedure described in our earlier report [26]. In short, gaseous mixture formed during the grinding process was subjected to a Balzer Thermostar mass spectrometer and ion current corresponding to $\mathrm{m} / \mathrm{z} 17$ (corresponds to $\mathrm{NH}_{3}{ }^{+}$) was monitored. While an increase in the ion current was observed after mixing of the CNTs and subsequent sampling, similar increase was observed for other background gases like nitrogen and oxygen. But, sampling after the grinding the CNTs showed further increase in the ion current for $\mathrm{NH}_{3}$ while increase in intensity for the background gases remained the same, proving formation of $\mathrm{NH}_{3}$ (Fig. 3d).Fig.3d, Ion current vs. time plots for $\mathrm{NH}_{3}{ }^{+}$obtained during the solid-state condensation reaction between $-\mathrm{OH}$ and $\mathrm{CONH}_{2}$ functionalized CNTs

The spectroscopic (Raman, FTIR and XPS) and mass spectral measurements are further confirmed using transmission electron microscopy. Fig. 4a shows bright field transmission electron microscope image of multi-walled carbon nanotubes used in current experiments. The diameter of these nanotubes varies from 40-100nm with a number of walls varying from 6-12 and the length of the nanotubes are in the order of few microns. There is no observable change observed in the size and morphology of the tubes due to functionalization. Fig. 4b shows bright field image of product produced due to reaction of chloride and $\mathrm{OH}$ functionalized nanotubes. It shows graphene with irregular morphology along with part of unzipped carbon nanotubes. These are stacked on each other in different orientation as shown in Fig.4c. On the other hand the bright field of $\mathrm{NH}_{2}$ and $\mathrm{OH}$ functional $\mathrm{CNT}$ shows graphene with smaller size and less number of 
walls. We observe less untreated CNTs. Based on the imaging, we have calculated percentage of graphene observed. To gain more insight about the reactivates of different combinations, we have used microscopy to find the yield range and size observed of graphene obtained in the $\mathrm{OH} /-$ $\mathrm{COOH}, \mathrm{OH} /-\mathrm{Cl}$ and $\mathrm{COOH} /-\mathrm{NH}_{2}$ combinations. The results are 40-60\%[400nm], 50-70\%[800 $\mathrm{nm}]$ and $60-80 \%[400 \mathrm{~nm}]$ respectively as shown in Fig. 4e-f. The yield results are compatible with the XPS data as well as chemical reaction discussed before.

In light of the above and according to the mechanism reportedelsewhere for reaction 1, the hydrogen-bond mediated proton transfer step in reaction 2 is followed by the formation of

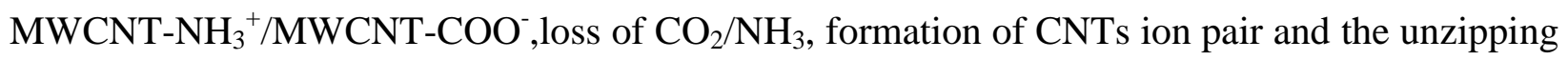
processes [26]. Also, dehydrochlorination in 3 is followed by decarboxylation of an ester group connecting $\mathrm{G}$ and $\mathrm{G}$ ' leading to ion-pairs formation and consequently CNTs unzipping. The higher yield of graphene via the acyl chloride CNT reaction as compared to that obtained via the mechano-chemical reaction of MWCNT-COOH and MWCNT-OH reported can be related to the high reactivity of the acyl chloride as compared to the carboxylic acid since the electronwithdrawing chlorine atom makes the carbonyl carbon more positive and therefore more susceptible to the attack by the hydroxyl group of MWCNT-OH. The higher yield in the $-\mathrm{NH}_{2} /-$ $\mathrm{COOH}$ reaction is due to the more exothermic proton transfer between the acidic carboxylic group and the basic amino group, a factor that will increase the \% of broken $\mathrm{C}-\mathrm{C}$ bonds in reaction 2 relative to that in 1 as we have confirmed in the detailed theoretical hot spots protocol of reaction 1 [33-36,26].

In conclusion, we have demonstrated the general case of a solid-state reaction between different functional groups covalently bound to carbon nanotubes that can be used to engineer different types of graphene. The exothermic reaction between these functionalities facilitated by simple 
mechanical grinding, results in bond breaking and the energy released in the process helps to break bonds and unzip the CNTs resulting in graphene. The hydrogen bond mediated proton transfer mechanism leading to the unzipping reaction of the MWCNTs can be used equally for different modifications of functional groups and therefore as a generic approach to develop synthetic frameworks for graphene productions such as doped graphene. The unzipping process is not accompanied by harsh physical and chemical techniques and accordingly can be used better in integration of graphene in electronic devices.

Acknowledgments: This work has been supported by U.S. Department of Defense: U.S. Air Force of Scientific Research for the Project MURI: "Synthesis and Characterization of 3-D Carbon Nanotube Solid Networks" Award No. FA9550-12-1-0035. Work at IIT Madras was supported by a grant through the Nano Mission, Government of India. Part of work was done while MAK was a visiting student at IIT Madras. CST would like to thank Indian Institute of Science, Bangalore for support. P.A.S.A., acknowledge financial support from the Brazilian Agencies CNPq, CAPES, and FAPESP and also thank the Center for Computational Engineering and Sciences at Unicamp for financial support through the FAPESP/CEPID Grant 2013/08293-7.

Author Contributions: MK and ATK proposed the project. MK and CST designed and conducted experiments. AS, KRK, SO, KK, KH, ACC helped in characterization. AS, KRK performed and TP supervised the mass spectral measurements and TP proposed the mechanochemical name for the reaction. MK, CST, RV, TP, ATK and PMA analyzed the data and wrote the paper. All authors discussed and revised the final manuscript.

\section{Competing financial interests}

The authors declare no competing financial interests. 


\section{References}

1. Vigdeman L, Zubarev ER. Therapeutic platforms based on gold nanoparticles and their covalent conjugates with drug molecules. Advanced Drug Delivery Reviews2013;65: 663-676.

2. Gibson J D.Khanal B P, Zubarev E. R.Paclitaxel-functionalized gold nanoparticles. J Am Chem Soc2007;129:11653.

3. Saha B, Bhattacharya J, Mukherjee A, Ghosh A, Santra C, Dasgupta A, Karmakar P. In vitro structural and functional evaluation of gold nanoparticles conjugated antibiotics. Nanoscale Res. Lett 2007;2:614-622.

4. Rai A, Prabhune A, Perry CC. Antibiotic mediated synthesis of gold nanoparticles with potent antimicrobial activity and their application in antimicrobial coatings. J Mater Chem2010;20:6789-6798.

5. Wang L, Zhu C, Wei X, Kan X. Preparation and application of functionalized nanoparticles of CdS as a fluorescence probe. Anal. Chim. Acta2005;468:35-41.

6. Shenhar R, Norsten TB, Rotello VM. Polymer-mediated nanoparticle assembly: Structural control and applications. Adv. Mater2005;17:657-669.

7. Jeng ES, Moll AE, Roy AC, Gastala JB, Strano MS. Detection of DNA hybridization using the near-infrared band-gap fluorescence of single-walled carbon nanotubes. Nano Lett2006;6:371-375.

8. Kovtyukhova NI, Mallouk TE, Pan L, Dickey EC. Individual single-walled nanotubes and hydrogels made by oxidative exfoliation of carbon nanotubes ropes. J. Am. Chem. Soc 2003; 125:9761-9769. 
9. Smith BM, Monthiooux M, and Luzzi DL. Encapsulated C60 in carbon nanotubes. Nature1998;396:323-325.

10. Zhu SE, Fei FL, and Wang GW. Mechanochemistry of fullerene and related materials. Chem.Soc. Rev2013;42:7535-7570.

11. Novoselov KS, et al. Electric field effect in atomically thin carbon films. Science2004; 306:666-669.

12. Qian M, et al. Formation of graphene sheets through laser exfoliation of highly ordered pyrolytic graphite. Appl Phys Lett2011;98:173108.

13. Zhao G, Shao D, Chen C, Wang, X. Synthesis of few-layered grapheme by hydrogen peroxide plasma etching of graphite. Appl Phys.Lett 2011;98:1831149.

14. Hernandez Y, et al. High-yield production of graphene by liquid-phase exfoliation of graphite. Nat Nanotechnol2008;3:563-568.

15. Jr Wsh, Offeman Jr WS H. R. E. Preparation of graphite oxide. J. Am. Chem. Soc1958;80:1339-1339.

16. Li D, Muller MB, Gilje S, Kaner RB, \& Wallace GG. Processable aqueous dispersions of graphene nanosheets. Nat Nanotechnol2008;3:101-10517.

17. Niyogi S, et al. Solution Properties of graphite and graphene. Journal of the American Chemical Society2006; 128:7720-7721.

18. Kim KS, et al. Large-scale pattern growth of graphene films for stretchable transparent electrodes. Nature2009;457:706-710.

19. Reina A, et al. Large area, few-layer graphene films on arbitrary substrates by chemical vapor deposition. Nano Lett2009;9:30-35. 
20. Wu Y, et al. Efficient and large-scale synthesis of few-layered graphene using an arcdischarge method and conductivity studies of the resulting films. Nano Res2010;3:661669

21. Berger $\mathrm{C}$, et al. Electronic confinement and coherence in patterned epitaxial graphene. Science2006;312:1191-1196.

22. Zhao J, et al. An approach for synthesizing graphene with calcium carbonate and magnesium. Carbon 2012;50:4939-4944.

23. Chakrabarti AC. Conversion of carbon dioxide to few-layer graphene. Journal of Materials Chemistry2011;21:9491.

24. Tour JM, et al. Longitudinal unzipping of carbon nanotubes to form graphene nanoribbons. Nature2009;458:872-876.

25. Jiao L, Zhang L, Wang X, Diankov G, \& Dai H. Narrow graphene nanoribbons from carbon nanotubes. Nature2009;458:877-880.

26. Kabbani, MA,et al. Ambient solid-state mechano-chemical reactions between functionalized carbon nanotubes. Nat. Commun2015;6:7291

27. Harris D, Bertolucci M. Symmetry and Spectroscopy New York, Dover Publications 1989

28. Yang D, GuoG, HuJ,Wang C, \& Jiang. Hydrothermal treatment to prepare hydroxyl group modified multi-walled carbon nanotubes, D.J. Mater. Chem 2008;18:350-354.

29. Ferrari AC, Basko DM. Raman spectroscopy as a versatile tool for studying the properties of graphene. Nature Nanotechnology2013;8:235-246

30. Ferrari AC,et al. Raman spectrum of graphene and graphene layers. Phys. Rev. lett2006; 97:187401-4. 
31. Graf D, et al. Spatially Resolved Raman Spectroscopy of Single and Few Layer Graphene.NanoLett2007;7:238-242

32. He R, et al. Observation of Low Energy Raman Modes in Twisted Bilayer Graphene.NanoLett 2013;13:3594-3601

33. Pauling J. General Chemistry, 3rd Ed, Freeman 1970:p.913.

34. Santos dos RPB, Perim E, Autreto PAS, Brunetto, and Galvão DS. On the unzipping of multiwalled carbon nanotubes. Nanotechnology 2012;23:465702.

35. Jund P, and Jullien R. Molecular-dynamics calculation of the thermal conductivity ofvitreous silica. Phys. Rev. B1999;59:13707-13711.

36. Chantrenne P, and Barrat JL. Finite Size Effects in Determination of Thermal Conductivities: Comparing Molecular Dynamics Results With Simple Models. J. HeatTransfer2004;126:577-585.

37. Carbon Nanotubes: Advanced Topics in the Synthesis, Structure, Properties and Applications. 2008, Springer

38. Chen MAJ, Hamon H, Hu Y, Chen AM, Rao PCEk, and Haddon RC. Science. 1998;282: 95.

\section{Figure Captions}

Figure 1. (a) General solid-state unzipping reaction of differently functionalized CNTs. (b)Unzipping double elimination reaction between MWCNT-COCl and MWCNT-OH: First elimination leads to the condensation of $\mathrm{HCl}$, which is followed by decarboxylation, formation of CNTs ion pair and consequent unzipping of CNTs by the heat of the exothermic reaction. (c) Unzipping double elimination reaction between MWCNT-COOH and $\mathrm{MWCNT}-\mathrm{NH}_{2}$ is 
mediated by hydrogen-bond formation, followed by proton transfer from $\mathrm{COOH}$ to $\mathrm{NH}_{2}$, formation of the $\mathrm{NH}_{3}$ and $\mathrm{CO}_{2}$, formation of CNTs ion pair and consequent unzipping of the CNTs by the heat of the exothermic reaction.

Figure 2. (a)ATR-IR of reactants and products in the solid-state reaction of MWCNT-NH $\mathrm{N}_{2}$ and MWCNT-COOH.Both the doublet band due to $\mathrm{NH}_{2}$ at about $3000 \mathrm{~cm}^{-1}$ and broad band in the 2500-3000 $\mathrm{cm}^{-1}$ region due to the stretching $\mathrm{O}-\mathrm{H}$ mode are absent in the reaction product in support of the elimination of $\mathrm{CO}_{2}$ and $\mathrm{NH}_{3}$.(b) ATR-IR of reactants and products in the solidstate reaction of MWCNT-OH and MWCNT-COCl. The conjugated carbonyl band at about $1750 \mathrm{~cm}^{-1}$ and broad band of the $\mathrm{O}-\mathrm{H}$ mode is absent in the product in support of $\mathrm{HCl}$ and $\mathrm{CO}_{2}$ elimination. $\mathrm{C}=\mathrm{C}$ band in the graphene product becomes dominant.(c-d) $\mathrm{C} 1 \mathrm{~s}$ XPS of reactants and products in the reactive combinations of $\mathrm{MWCNT}-\mathrm{NH}_{2} / \mathrm{MWCNT}-\mathrm{COOH}$ (c), and MWCNT-OH/ MWCNT-COCl(d).The signal at $289.2 \mathrm{eV}$ due to the carbonyl group in both combinations disappears upon grinding of $\mathrm{CNTs}$ and is dominated by the $\mathrm{C}=\mathrm{C}$ signal of graphene at $284.6 \mathrm{eV}$.The higher intensity of the latter band in the $\mathrm{NH}_{2} / \mathrm{COOH}$ combination is compatible with the bigger yield of this combination relative to the $\mathrm{COCl} / \mathrm{OH}$ combination.(e-f) Raman spectra of MWCNT-NH 2 /MWCNT-COOH,(e), and MWCNT-OH/ MWCNT$\mathrm{COCl}$,(f).The G,D and 2Dbands are all blue shifted in the graphene product relative to those of CNTs.

Figure 3: A simple schematic of the experimental set up used for the detection of evolved $\mathrm{HCl}$ gas (A), SEM images of the lumps of $\mathrm{AgCl}$ particles formed (B) at low magnification (a) and high magnification (b), EDAX data of the $\mathrm{AgCl}$ formed (C). In (C), (a) is the SEM image of one of the lumps of $\mathrm{AgCl}$ formed, and elemental mapping of the same lump collecting the intensities 
of $\mathrm{Ag} \mathrm{L} \mathrm{(a)} \mathrm{and} \mathrm{Cl} \mathrm{K}$ (b). Elemental composition obtained from the EDAX analysis is shown in the inset.

Figure 4. (a) Low magnification bright field TEM image of multiwalled CNTs, high magnification images revealing number of walls shown in the inset. (b) Low magnification bright field image of graphene produced after reaction (c) SAD pattern from these products (d) The graphene produced during $\mathrm{Cl}$ functionalization (e) \%yield produced in $\mathrm{OH}, \mathrm{NH} 2$ and $\mathrm{Cl}$ functionalized using current work (f) Size of particle produced in current methods.

Table 1:Different modifications of the mechanochemical reaction between differently functionalized MWCNTs

\begin{tabular}{|l|l|}
\hline Reaction & Types of elimination \\
\hline $\mathbf{1}$ & Water condensation +decarboxylation \\
\hline $\mathbf{2}$ & Dehydroamination + decarboxylation \\
\hline $\mathbf{3}$ & Dehydrochlorination+decarboxylation \\
\hline
\end{tabular}

Table 2: Yield and $I_{2 D} / 1_{G}$ ratios of the graphene obtained via reactions of different functionalities CNTs.

\begin{tabular}{|c|c|c|}
\hline Solid-state reaction & Yield & $\mathbf{I}_{2 D} / \mathbf{I}_{G}$ \\
\hline$-\mathrm{COOH} / \mathrm{OH}$ & $60 \%{ }^{26}$ & 1.20 \\
\hline$-\mathrm{COOH} / \mathrm{NH} 2$ & $75 \%$ & 0.95 \\
\hline$-\mathrm{COCl} / \mathrm{OH}$ & $71 \%$ & 2.10 \\
\hline
\end{tabular}


GENERAL PROCESS

DIFFERENT FUNCTIONALIZATIONS

(a)
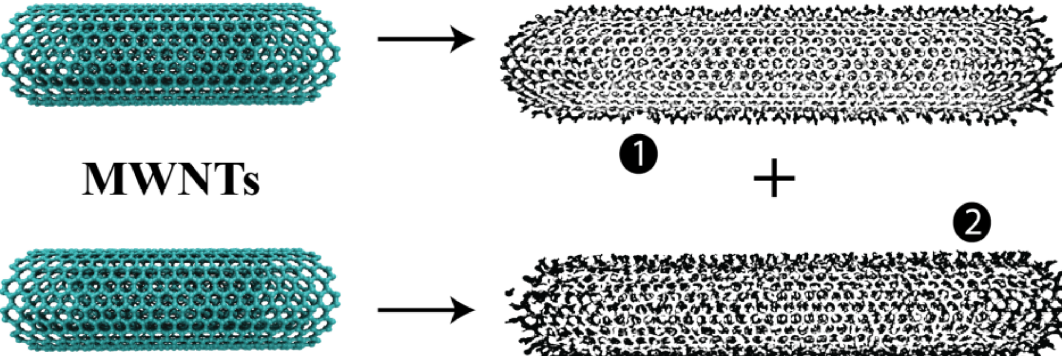

(b)

$1+$

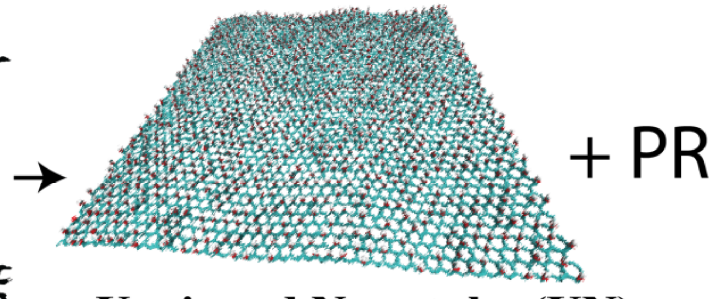

Unzipped Nanotube (UN)

(1) COCI-CNT

2 OH-CNT

CASE A

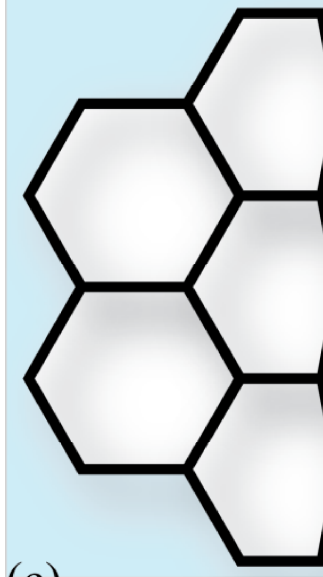

(c)
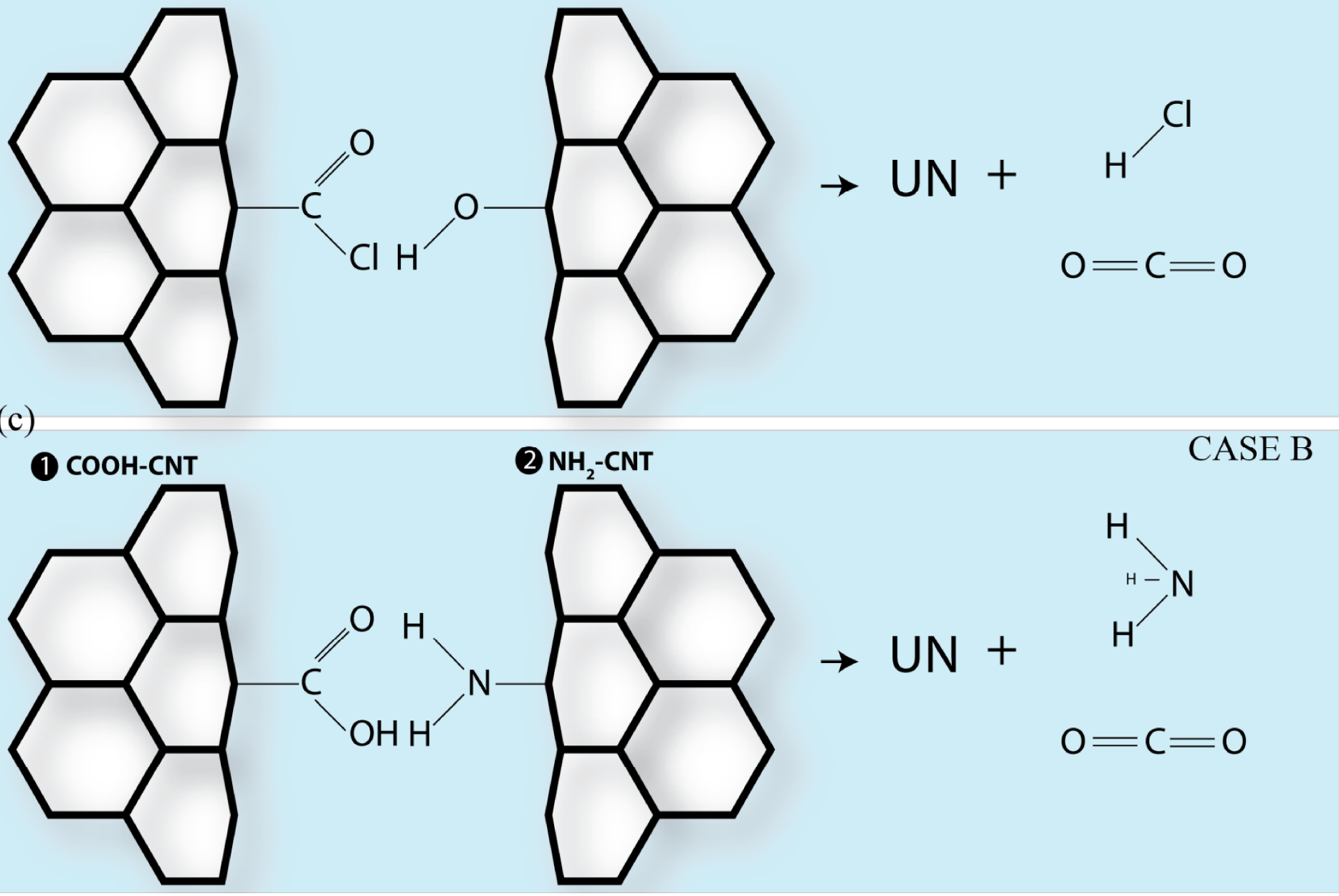

Fig.1 
(a)

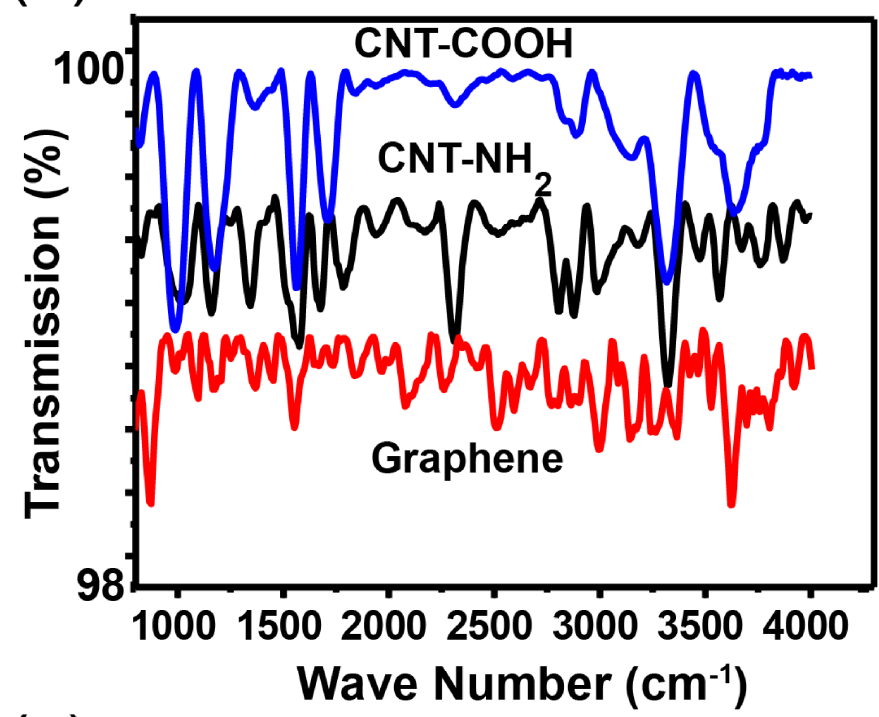

(c)

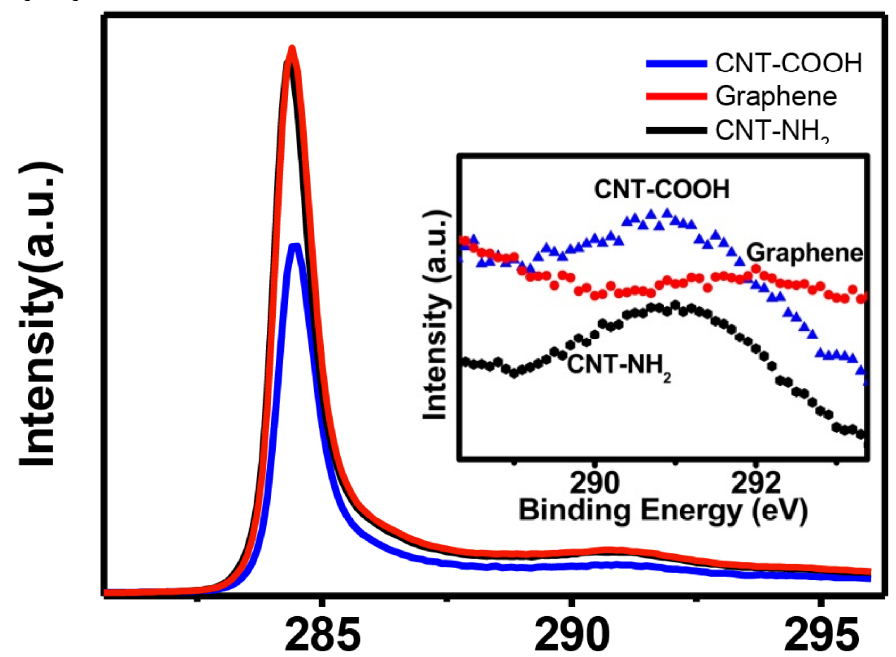

(e)

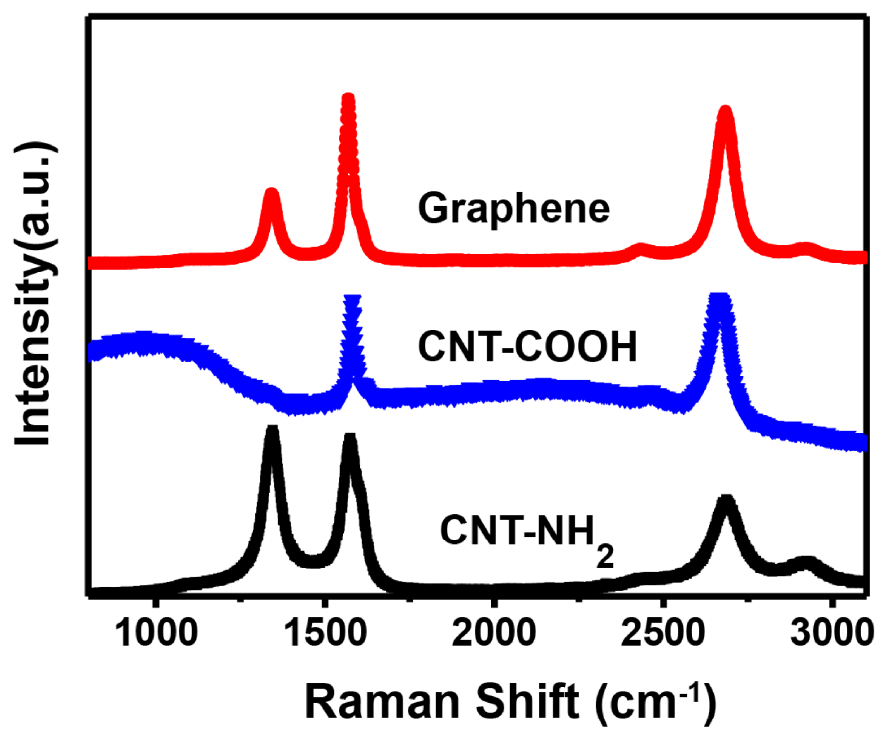

(b)

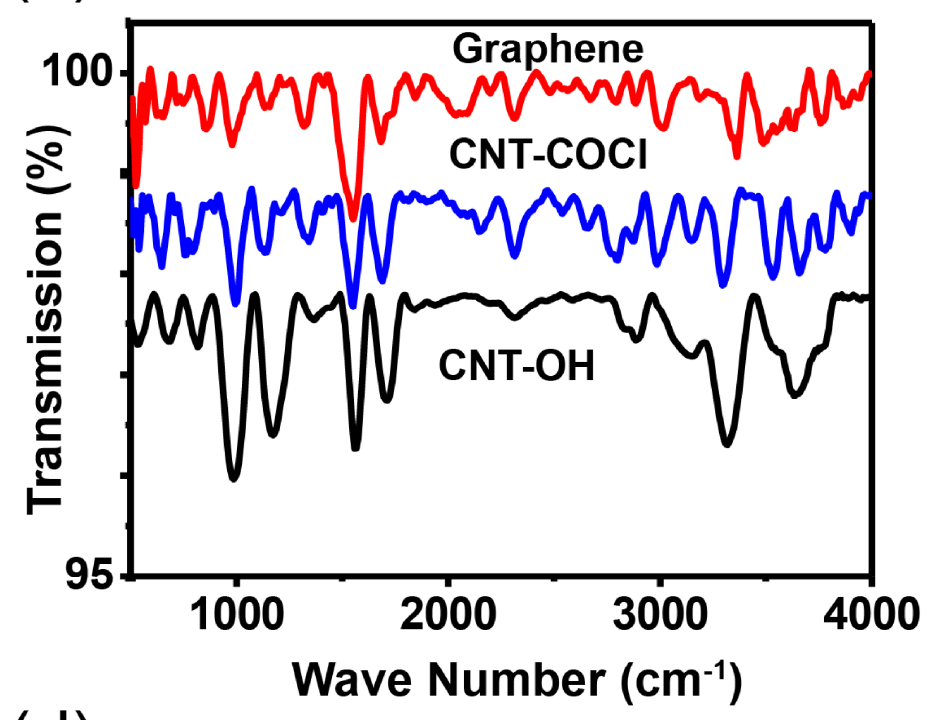

(d)

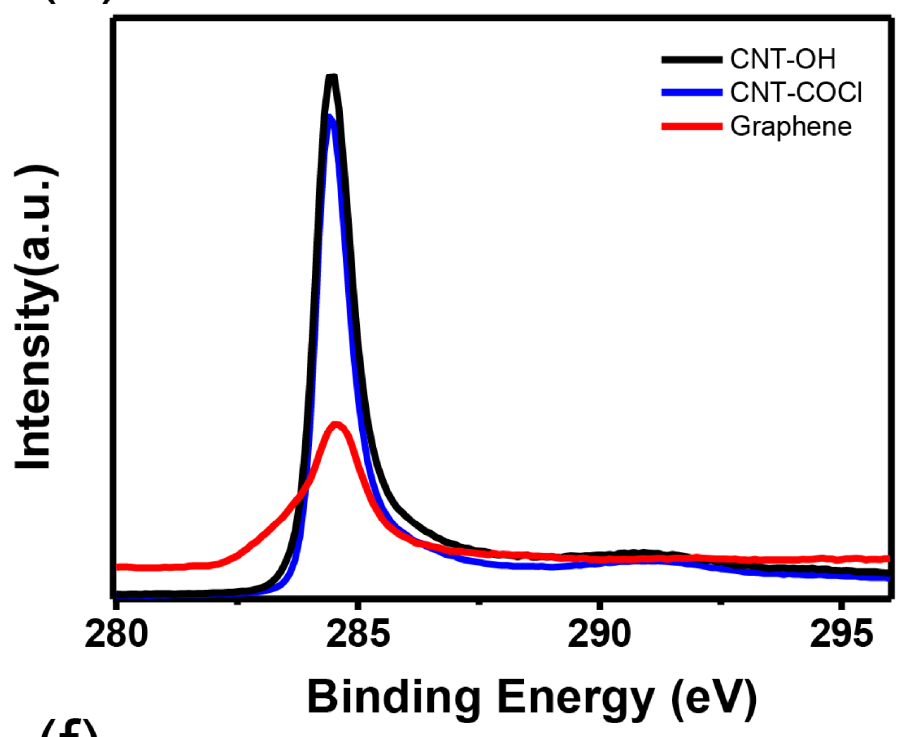

(f)

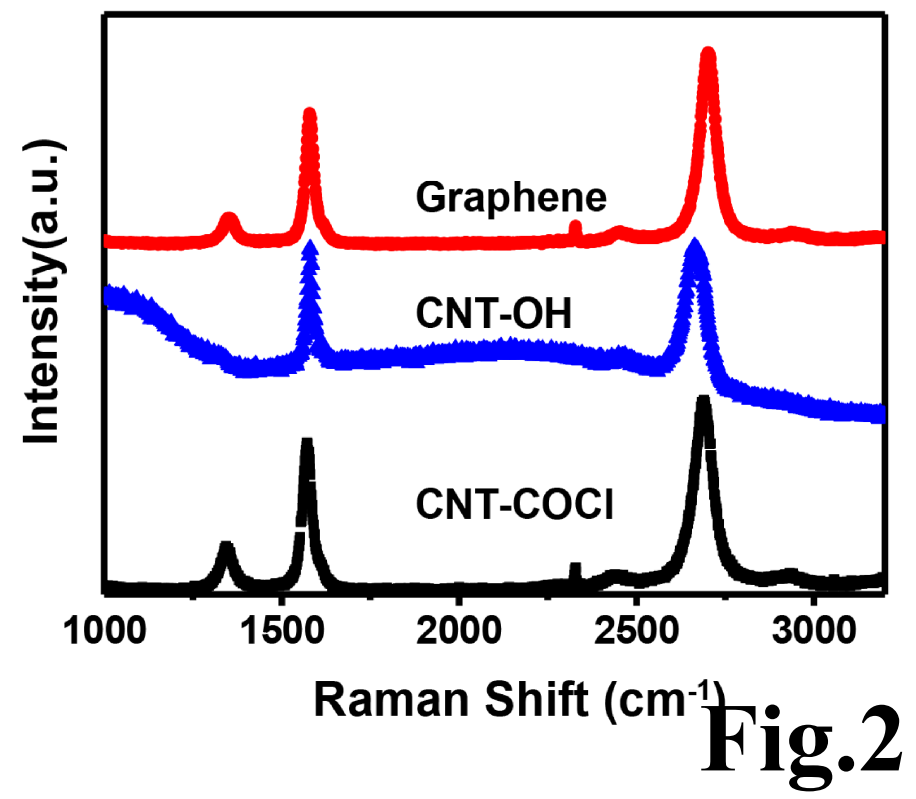




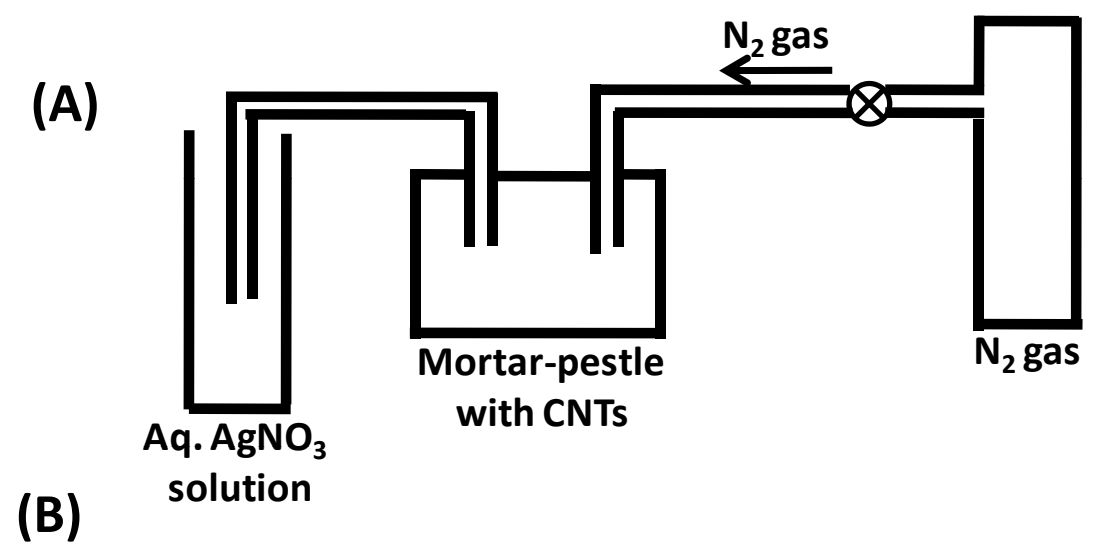

(C)
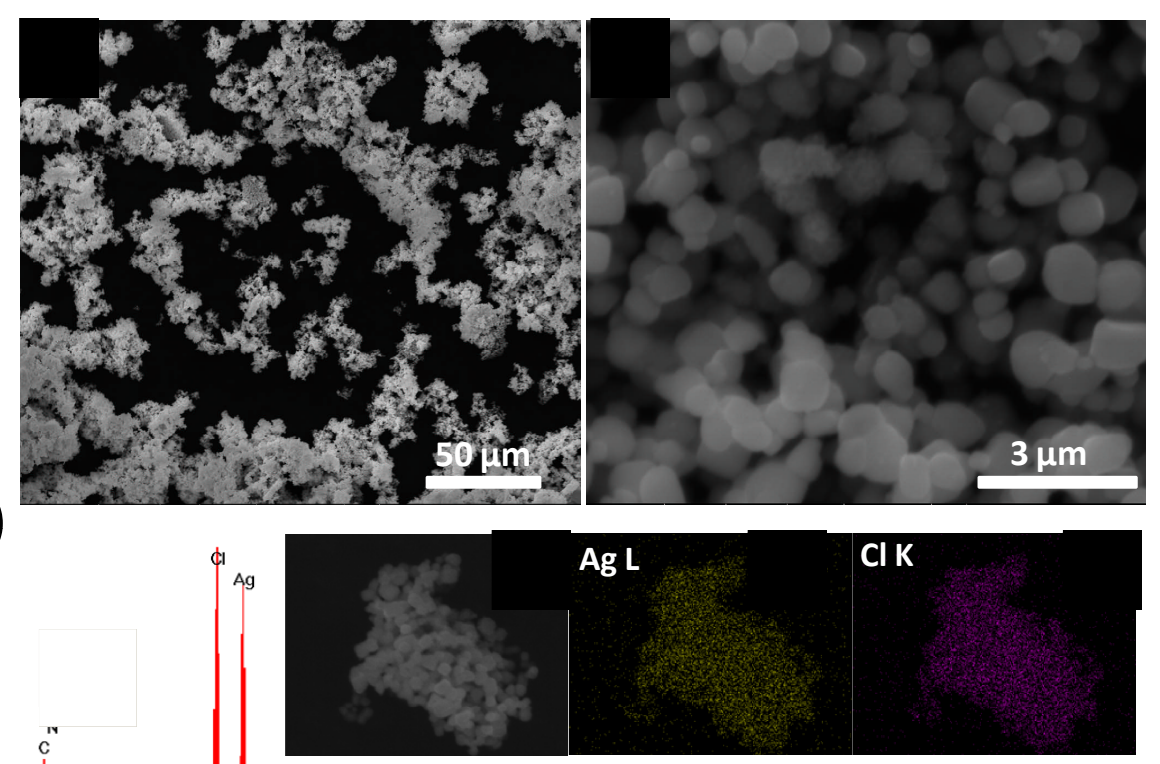

Elem Wt \% At \%

Cl K 24.0949 .13

Ag L 75.9150 .87

Total 100.00100 .00

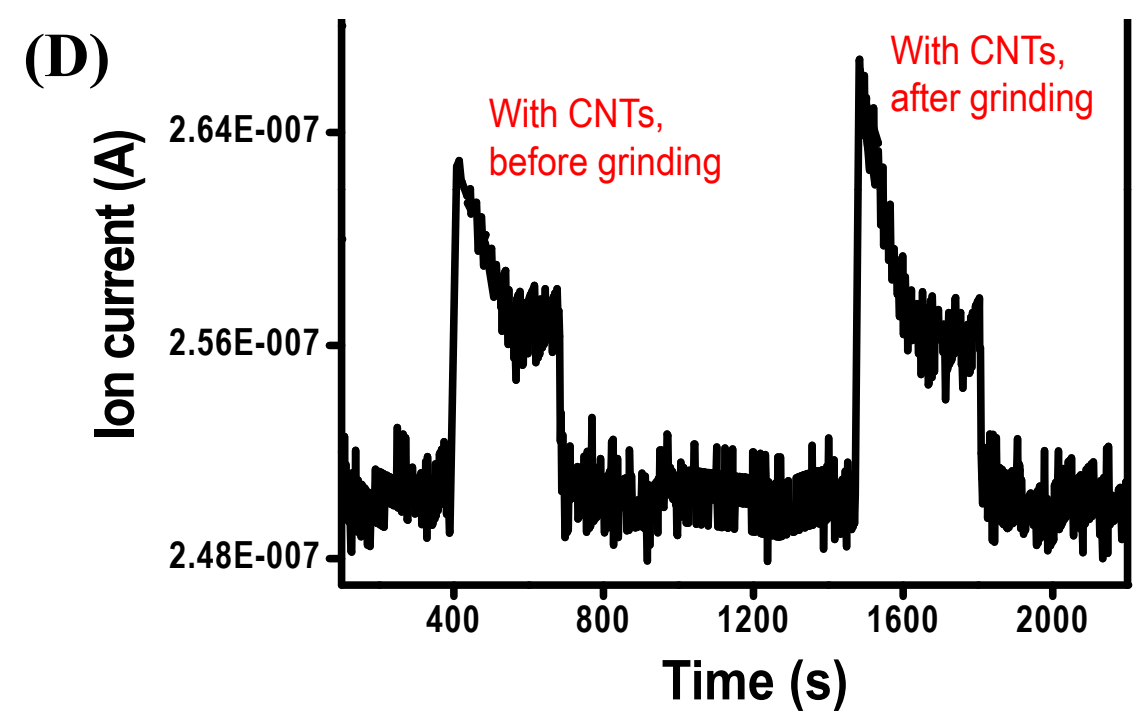



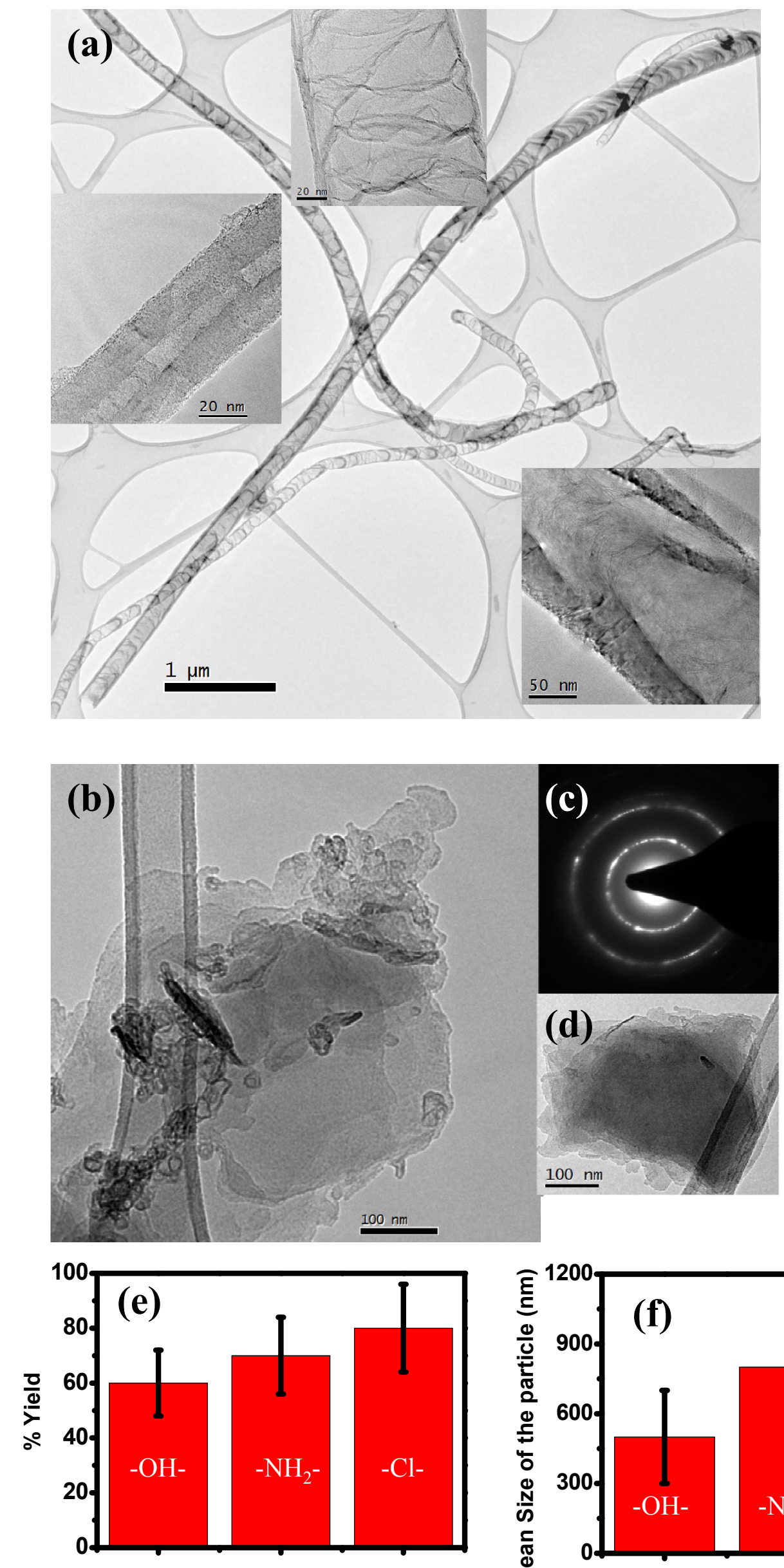

Fig.4

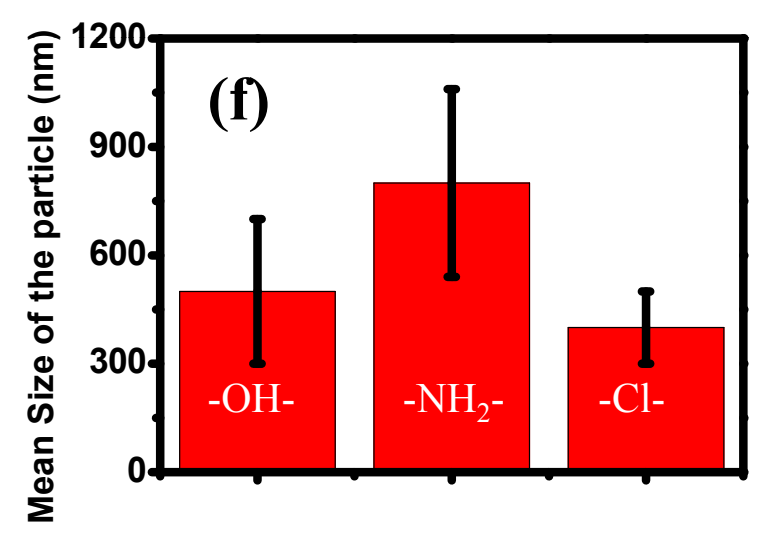

